\title{
RURAL SPRING GEOMETER MOTHS (LEPIDOPTERA, GEOMETROIDEA LEACH, 1815) IN KOSOVO AND METOHIA (SERBIA)
}

\author{
NEBOJŠA V. ŽIVIĆ ${ }^{*}$, PREDRAG N. JAKŠIĆ ${ }^{1}$ \\ ${ }^{1}$ Faculty of Natural Sciences and Mathematics, University of Priština - Kosovska Mitrovica, Serbia
}

\begin{abstract}
This paper shows the presence of the rural geometer moths in 15 localities of Kosovo and Metohia (Serbia). A review of existing data regarding the area of Kosovo and Metohia is given. Through research, the presence of 48 species was revealed. Between them, five species are reported as new for the fauna of Kosovo and Metohia: Plagodis pulveraria (Linnaeus, 1758), Scotopteryx luridata (Hufnagel, 1767), Catarhoe cuculata (Hufnagel, 1767), Anticlea badiata (Denis \& Schiffermüller, 1775) and Anticlea derivata (Denis \& Schiffermüller, 1775).
\end{abstract}

Keywords: Lepidoptera, Geometridae, Kosovo and Metohia (Serbia).

\section{INTRODUCTION}

The order Lepidoptera Linnaeus1758, with 45 superfamilies, represents one of the larger order of insects. Among them, the superfamily of geometer moths (Geometroidea Leach, 1815), with 999 species in Europe, belongs to the number of species in the more significant group of Lepidoptera (Nieukerken et al., 2011). In Serbia, this group of insects is represented by more than 350 species (Dodok, 2006). With this in mind, it is clear that the fauna of this group is insufficiently explored in Serbia.

The first faunistic data on the geometer moths in Kosovo and Metohia are found in the work of Rebel (1917). At that time, during the First World War, part of Metohija was connected to Montenegro ("Neumontenegro").The area of Žljeb Mt., Novo Selo (north of Peć) and the surroundings of Peć, Ribarić and Čečevo Brdo were investigated. The presence of 95 species was established and this result represents a pioneering contribution of the author.

Vukčević (1954) published next data on the geometer moths in Kosovo and Metohia. Among the identified species of plant diseases and pests, the author also lists the winter moths specie Operophtera brumata L. and Erannis defoliaria (Clerck, 1759). Đorđije Đorović, from the Forestry Institute in Peć, specialized in studying the defoliators of oak. Among the numerous representatives of defoliators, Đorović identified 25 species of Geometridae (Đorović, 1974, 1976, 1979, 1980 and 1992).

After this period, practically no one specialized in the study of geometer moths in Kosovo and Metohia. In the period from 1972 to 1999 , Predrag Jakšić collected specimens of this group. Some of the data are published (Jakšić, 2016; Jakšić \& Ristić (1999) 2001). (Tomić et al., 2002). One part of his data, comprising 80 species, is included in a study of the family of this group in Serbia (Tomić et al., 2002). In addition, several other authors have contributed to the knowledge of this group, as indicated in the References.

Some of rural and urban Geometridae species were primarily known for melanism, an example of a wellinvestigated species of the genus Biston (Bishop, 1972; Ford, 1955). Therefore, they can be indicators of the quality of the environment. Considering Rebel's (1917) research is already 100 years old, it was interesting to compare his results with ours.

As can be seen from this review, the fauna of this group of Lepidoptera is still insufficiently studied in Serbia. Likewise, the fauna of urban and rural areas, which usually contains synanthropic species, has remained under-researched. In addition, previous research was carried out for the most part during the summer period.

Bearing these facts in mind, we thought it would be important to study the urban and rural spring fauna of geometer moths in a few localities of Kosovo and Metohia.

\section{MATERIALS AND METHODS}

Specimens of the studied species were collected in the territory of Kosovo and Metohia in a period from 1974 to 2019. Collection was carried out at the following sites (Tab. 1).

Altitude and site coordinates are determined according to Google Earth, available on the Internet.

Collection was carried out at night, using a $160 \mathrm{~W}$ bulb (Mercury vapour Lamp, 160 W, BB Link Lighting). Preparation of the collected material and the production of permanent microscope slides of the genital armatures were carried out by standard procedure. Genital armatures were recorded as needed on a Nikon binocular microscope using a digital Nikon camera with an AF-S Micro Nikkor Lens.

Species determination was based on wing parameters and analysis of genital armatures, if necessary. The literature given in

\footnotetext{
* Corresponding author: nebojsa.zivic@pr.ac.rs 
the References was used for determination based on the wing parameters.

The classification of Lepidoptera at the levels of order, superfamily and family is according to Nieukerken et al. (2011). Taxonomy and nomenclature of examined species was done according to Müller et al. (2019). Species ID numbers and systematic order are done according to Karsholt \& Razowski (1996). Species determination was carried out on the basis of contemporary literary sources.

Abbreviations: $\mathrm{m}$ - Male, $\mathrm{f}-$ Female; $\mathrm{K} \& \mathrm{M}-$ Kosovo \& Metohia (Косово и Метохија, Србија).

Tabela 1. List of examined places.

\begin{tabular}{|c|c|c|c|}
\hline \multirow[t]{2}{*}{ LOCALITY } & \multirow{2}{*}{$\begin{array}{l}\text { ELEVATION } \\
\text { (m) }\end{array}$} & \multicolumn{2}{|c|}{ COORDINATES } \\
\hline & & Latitude $\varphi(\mathrm{N})$ & Longitude $\lambda(\mathrm{E})$ \\
\hline Bostane (Novo Brdo) & 860 & $42^{\circ} 36^{\prime} 47^{\prime \prime}$ & $21^{\circ} 25^{\prime} 33^{\prime \prime}$ \\
\hline Gornje Kusca (Gnjilane) & 556 & $42^{\circ} 29^{\prime} 48^{\prime \prime}$ & $21^{\circ} 28^{\prime} 56^{\prime \prime}$ \\
\hline Leposavić & 508 & $43^{\circ} 06^{\prime} 10^{\prime \prime}$ & $20^{\circ} 48^{\prime} 30^{\prime \prime}$ \\
\hline Parteš (Gnjilane) & 490 & $42^{\circ} 24^{\prime} 07^{\prime \prime}$ & $21^{\circ} 26^{\prime} 01^{\prime \prime}$ \\
\hline Pasjane (Gnjilane) & 500 & $42^{\circ} 24^{\prime} 25^{\prime \prime}$ & $21^{\circ} 29^{\prime} 44^{\prime \prime}$ \\
\hline Pridvorica & 580 & $42^{\circ} 55^{\prime} 22^{\prime \prime}$ & $20^{\circ} 41^{\prime} 21^{\prime \prime}$ \\
\hline Priština, Gazimestan & 630 & $42^{\circ} 41^{\prime} 20^{\prime \prime}$ & $21^{\circ} 07^{\prime} 46^{\prime \prime}$ \\
\hline Priština, Grmija & 700 & $42^{\circ} 40^{\prime} 30^{\prime \prime}$ & $21^{\circ} 11^{\prime} 57^{\prime \prime}$ \\
\hline Sevce (Šar-Planina) & 1060 & $42^{\circ} 12^{\prime} 48^{\prime \prime}$ & $20^{\circ} 56^{\prime} 22^{\prime \prime}$ \\
\hline Stanišor (Gnjilane) & 548 & $42^{\circ} 29^{\prime} 30^{\prime \prime}$ & $21^{\circ} 27^{\prime} 43^{\prime \prime}$ \\
\hline Štrpce (Šar-Planina) & 890 & $42^{\circ} 14^{\prime} 19^{\prime \prime}$ & $21^{\circ} 01^{\prime} 45^{\prime \prime}$ \\
\hline Velji Breg (Zubin Potok) & 630 & $42^{\circ} 55^{\prime} 54^{\prime \prime}$ & $20^{\circ} 40^{\prime} 41^{\prime \prime}$ \\
\hline Vrbeštica (Šar-Planina) & 1011 & $42^{\circ} 14^{\prime} 34^{\prime \prime}$ & $20^{\circ} 58^{\prime} 32^{\prime \prime}$ \\
\hline Zubin Potok & 567 & $42^{\circ} 54^{\prime} 52^{\prime \prime}$ & $20^{\circ} 41^{\prime} 23^{\prime \prime}$ \\
\hline Zvečan & 583 & $42^{\circ} 54^{\prime} 24^{\prime \prime}$ & $20^{\circ} 50^{\prime} 35^{\prime \prime}$ \\
\hline
\end{tabular}

\section{RESULTS: LIST OF EXAMINED SPECIES}

Superfam. Geometroidea Leach, 1815

Fam. Geometridae Leach, 1815

7530 Ligdia adustata ([Denis \& Schiffermüller], 1775): Pridvorica, 5. May 2018, 1m; Velji Breg, 13. April 2018., 1m; Zubin Potok, 16. April 2018., 1m. New species for Serbia.

7540 Macaria alternata ([Denis \& Schiffermüller], 1775): Gornje Kusce, 16. May 2018., 1m; Štrpce, 24. June 2018. 1f.

7581 Neognopharmia stevenaria (Boisduval, 1840): Pasjane, 19. April 2018., $1 \mathrm{~m}$.

7606 Plagodis pulveraria (Linnaeus, 1758): Gornje Kusce, 4. May 2018., $1 \mathrm{f}$.

7613 Opisthograptis luteolata (Linnaeus, 1758): Štrpce, 20. May 2018., $1 \mathrm{~m}$.

7618 Therapis flavicaria ([Denis \& Schiffermüller], 1775): Pridvorica, 5. May 2018., $1 \mathrm{~m}$.

7622 Eilicrinia cordiaria (Hübner, 1790): Gnjilane, Gornje Kusce, 7. May 2018., Genitalia checked, slide SR-3120 (Fig. 1).

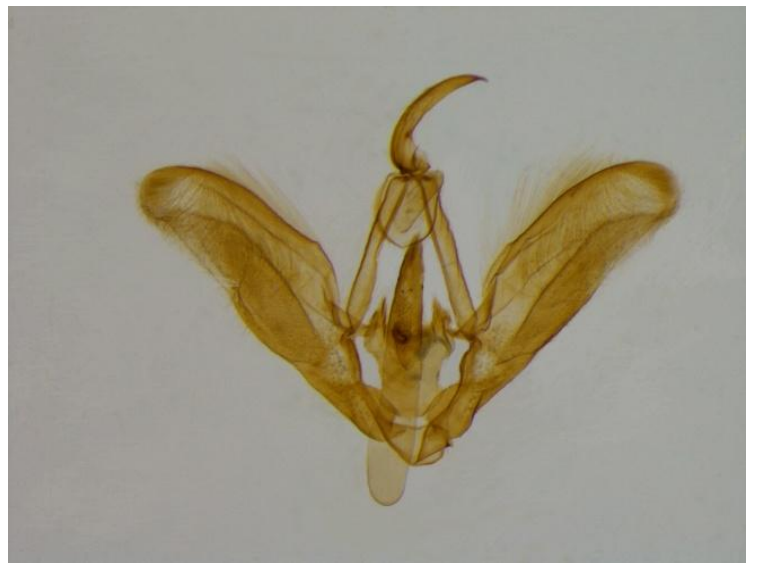

Figure 1. E. cordiaria (Hübner, 1790): Gnjilane, Gornje Kusce, 7. May 2018., Male genitalia.

7636 Ennomos erosaria ([Denis \& Schiffermüller], 1775): Leposavić, 26. May 2018., $1 \mathrm{~m}$ Genitalia checked, slide SR-3135 (Figs. 2a \& 2b).

7642 Selenia lunularia (Hübner, [1788]): Gornje Kusce, 8. April, 2018., 1m; Pasjane, 4. April 2018., $1 \mathrm{f}$.

7650 Dasycorsa modesta (Staudinger, 1879): Gnjilane, Gornje Kusce, 15. Mart 2018., 1m; 30. Mart 2018., 1m; 10.-16. 
April 2018., 3m; Gnjilane, Stanišor, 5.-22. April 2018., 3m, Genitalia checked. (Figs. 3a \& 3b).

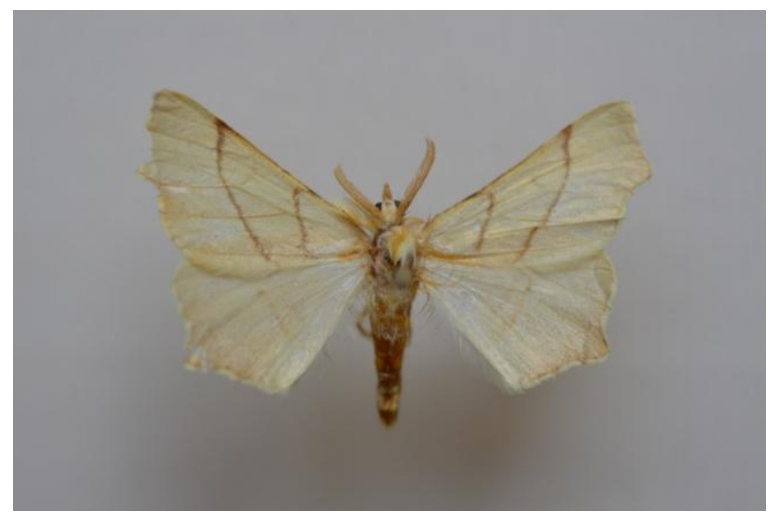

Figure 2a. E. erosaria ([Denis \& Schiffermüller], 1775): Leposavić, 26. May 2018., Male adult.

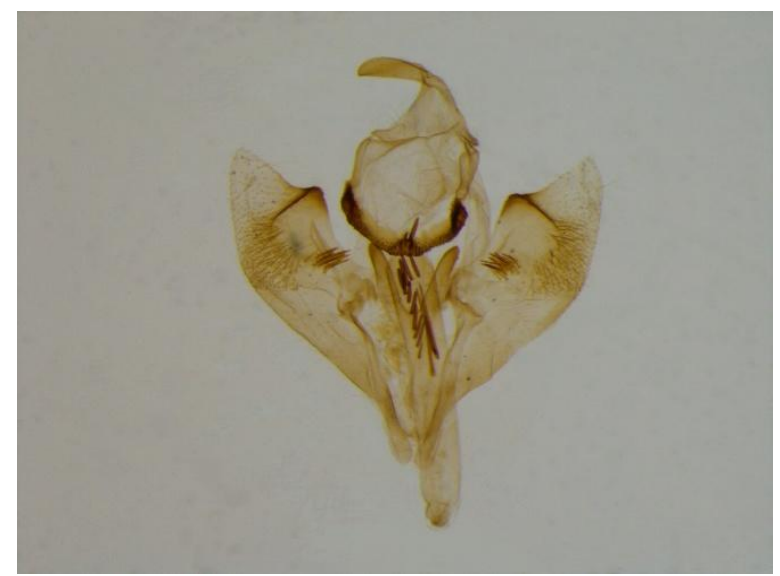

Figure 2b. E. erosaria ([Denis \& Schiffermüller], 1775): Leposavić, 26. May 2018., Male genitalia.

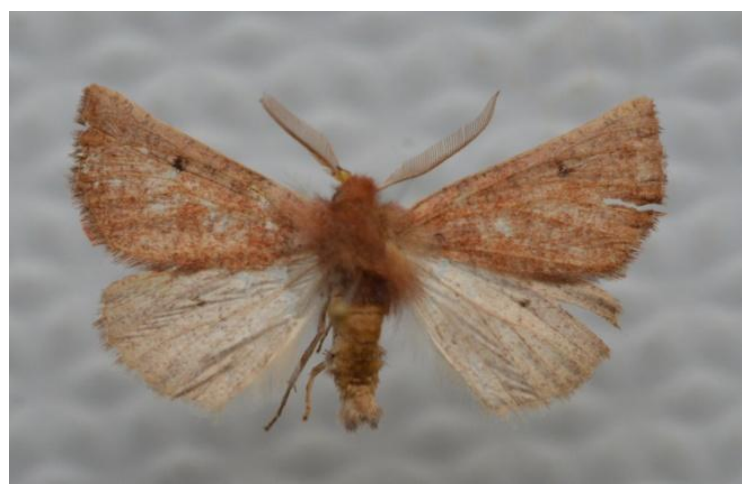

Figure 3a. D. modesta (Staudinger, 1879): Gnjilane, Gornje Kusce, 16. April 2018. Male, adult.

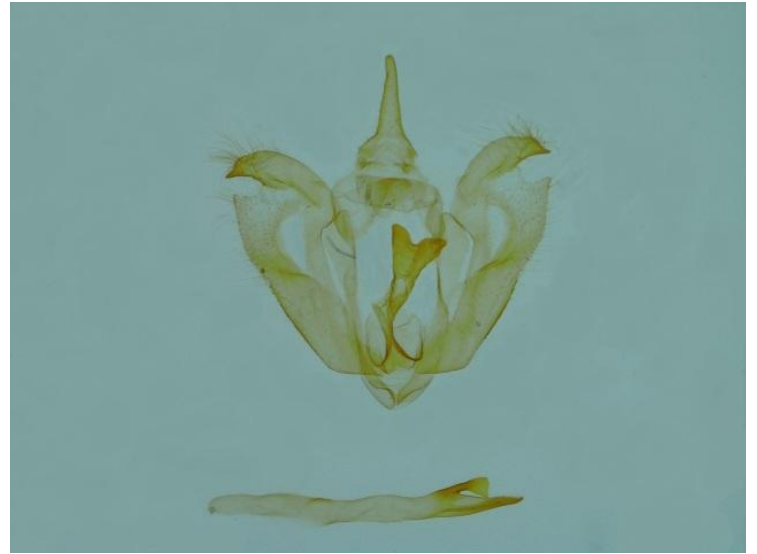

Figure 3b. D. modesta (Staudinger, 1879): Gnjilane, Gornje Kusce, 30. Mart 2018. Male, genitalia.

7661 Asovia maeoticaria (Alphéraky, 1876): Gnjilane, Gornje Kusce, 13. April 2018., 1f. So far, for Serbia this species reported Djuric \& Hric (2013) and Beshkov (2015). New species for $\mathrm{K} \& \mathrm{M}$.

7663 Colotois pennaria (Linnaeus, [1760]): Sevce, 20. October, $1 \mathrm{~m}$; Štrpce, 18. October 2018., 1f. So far, Đorović (1976, 1992) reported this species for K \& M.

7671 Apocheima hispidaria ([Denis \& Schiffermüller], 1775): Gornje Kusce, 2. February 2018, 1 m; 17. February 2018., 1m; 15. March 2018., 1m. Đorović (1976 \& 1979) reported this species for $\mathrm{K} \& \mathrm{M}$.

7672 Phigalia pilosaria ([Denis \& Schiffermüller], 1775): Vrbeštica, 1. March 2018., 4m. Genitalia checked, slide SR-3125 (Figs.4a \& 4b). Đorović (1976, 1979, 1992) reported this species for $\mathrm{K} \& \mathrm{M}$.

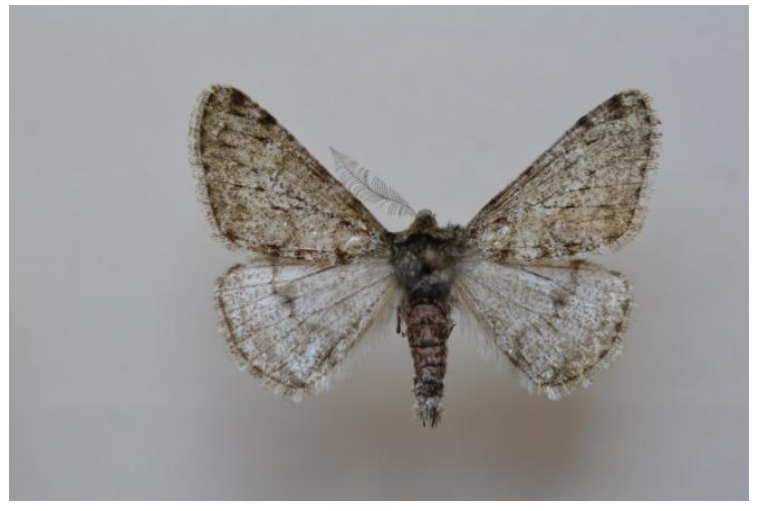

Figure 4a. P. pilosaria ([Denis \& Schiffermüller], 1775). ŠarPlanina, Vrbeštica, $1011 \mathrm{~m}, 1$. Mart, 2019. Male adult. 


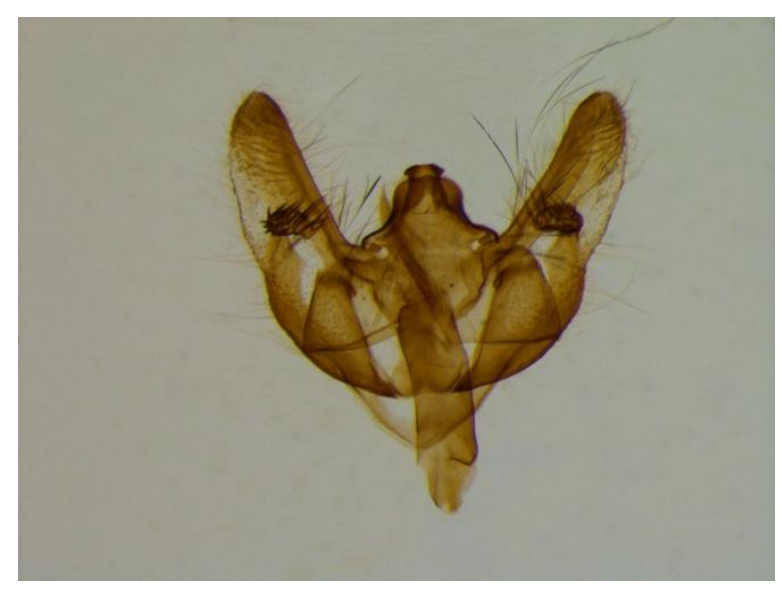

Figure 4b. $P$. pilosaria ([Denis \& Schiffermüller], 1775). ŠarPlanina, Vrbeštica, $1011 \mathrm{~m}, 1$. Mart, 2019. Male genitalia.

7674 Lycia hirtaria (Clerck, 1759): Štrpce, 29. April 2018., $1 \mathrm{~m}$.

7676 Lycia graecarius (Staudinger, 1861): Gornje Kusce, 19.

March 2018, 1m; Velji Breg, 30. March 2018, 1m. Jakšić (2016; 2018) and Jakšić \& Ristić (1999) reported this species for $\mathrm{K} \& \mathrm{M}$.

7685 Biston strataria (Hufnagel, 1767): Gornje Kusce, 5. March 2018., 1m; 29. March 2018., $1 \mathrm{~m}$.

7694 Agriopis bajaria ([Denis \& Schiffermüller], 1775): Štrpce,

25. October 2018., $1 \mathrm{~m}$. This is the second data for this species on K \& M, so far Đorović (1979) reported it for Dulje and Birač.

7695. Agriopis aurantiaria (Hübner, 1799): Stanišor, 31. September 2018., 1 m.; Štrpce, 25. October 2018., $1 \mathrm{~m}$.

7696 Agriopis marginaria (Fabricius, [1777]): Vrbeštica, 1. March 2018., 2m; Štrpce, 29. April 2018., 1m; Priština, Grmija, 6. March 1979., 1m; 20. March 1980., $1 \mathrm{~m}$. Genitalia checked, slides SR-3124 \& SR-3126.

7699 Erannis defoliaria (Clerck, 1759): Zvečan, 26. October 2018., 1m; Bostane (Novo Brdo), 27. November 1982., $1 \mathrm{~m} ; 10$. December 1982., 1m. Genitalia checked, slides SR-3128 and SR-3129. (Fig. 5).

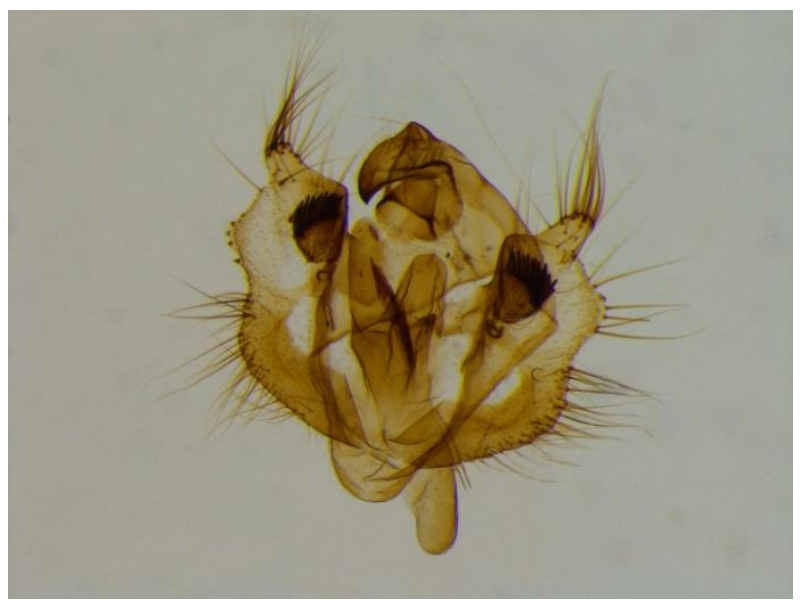

Figure 5. E. defoliaria (Clerck, 1759), Bostane (Novo Brdo), 10. December 1982. Male genitalia.
7804 Ematurga atomaria (Linnaeus, 1758): Velji Breg, 9. May 2018., $1 \mathrm{~m}$.

7824 Cabera pusaria (Linnaeus, 1758): Parteš, 12. May 2018., $1 \mathrm{f}$.

7826 Cabera exanthemata (Scopoli, 1763): Štrpce, 20 May 2018., $1 \mathrm{~m}$.

7831a Aleucis orientalis (Staudinger, 1892): Priština, Gazimestan, 9. April 1974., 1f.; Štrpce, 29.April 2018., $1 \mathrm{~m}$.

7916 Siona lineata (Scopoli, 1763): Gornje Kusce, 7. May 2018., 1m; Zubin Potok, 15. May 2018., 1f.

7953 Alsophila aescularia ([Denis \& Schiffermüller], 1775): Gornje Kusce, 17. March 2018., 2m; Strpce, 29. April 2018., 3m; Vrbeštica 1. March 2018., $5 \mathrm{~m}$.

7971 Comibaena bajularia ([Denis \& Schiffermüller], 1775): Leposavić, 24. May 2018., $1 \mathrm{~m}$ Genitalia checked, slide SR-3136 (Figs. 6a \& 6b).

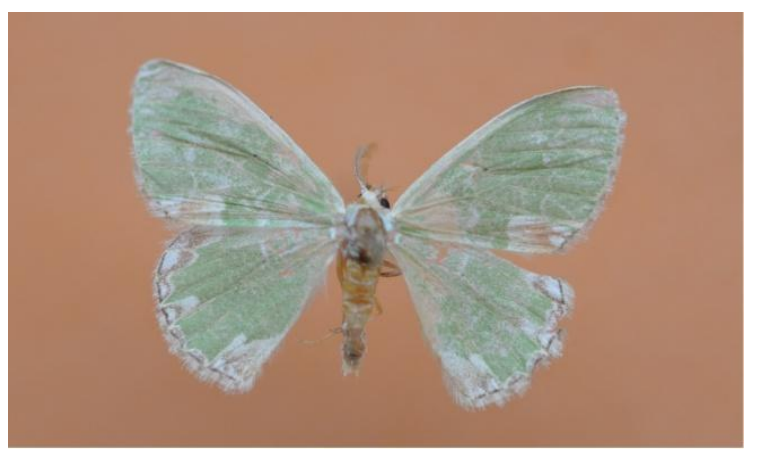

Figure 6a. C. bajularia ([Denis \& Schiffermüller], 1775): Leposavić, 24. May 2018., Male adult.

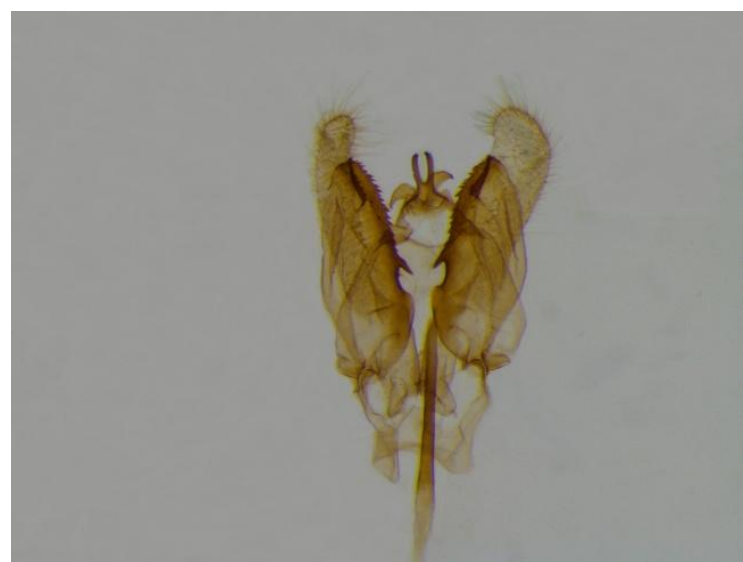

Figure 6b. C. bajularia ([Denis \& Schiffermüller], 1775): Leposavić, 24. May 2018., Male genitalia.

8000 Hemistola chrysoprasaria (Esper, 1795): Gornje Kusce, 20. May 2018., $1 \mathrm{f}$.

8020 Cyclophora quercimontaria (Bastelberger, 1897): Pridvorica, 7. May 2018., $1 \mathrm{~m}$ and 1f.

8024 Cyclophora linearia (Hübner, [1799]): Pridvorica, 5. May, 2018., 1m; Zubin Potok, 15. May 2018, $1 \mathrm{f}$. 
8028 Timandra comae A. Schmidt, 1931: Pridvorica, 5. May 2018, $1 \mathrm{~m} \&$ 7. May 2018., $1 \mathrm{~m}$.

8036 Scopula immorata (Linnaeus, 1758): Parteš, 10 May 2018., 1f.

8045 Scopula ornata (Scopoli, 1763): Šar-Planina Mt., Štrpce, 20. May 2019., 1m. Genitalia checked, slide SR-3116 (Fig. 7).

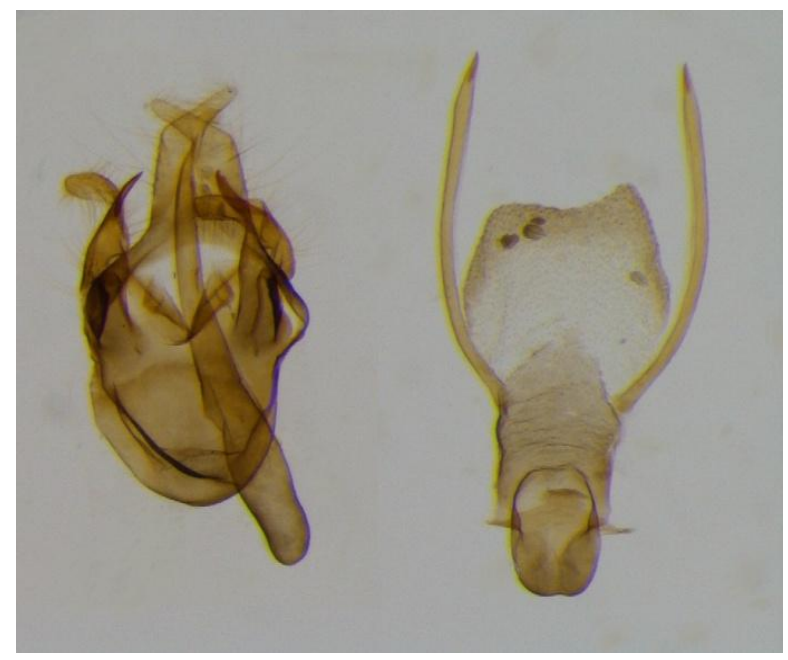

Figure 7. S. ornata (Scopoli, 1763): Šar-Planina Mt., Štrpce, 20. May 2019. Male genitalia.

8051 Scopula decorata ([Denis \& Schiffermüller], 1775): Pasjane, 6. June 2018., 1f. (Fig. 8). Genitalia checked, slide SR-3117.

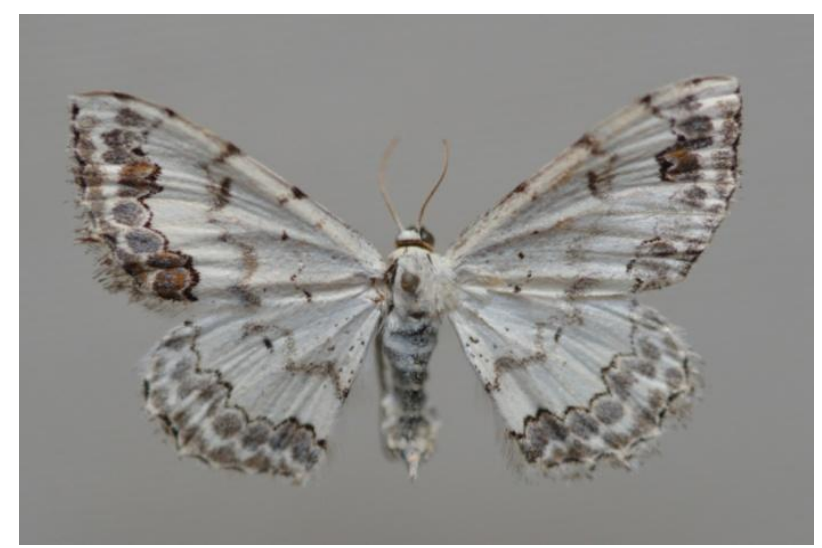

Figure 8. S. decorata ([Denis \& Schiffermüller], 1775): Pasjane, 6. June 2018., Female, adult.

8059 Scopula marginepunctata (Goeze, 1781): Pridvorica, 9. May 2018., 1m. Genitalia checked, slide SR-3121.; ŠarPlanina, Štrpce, 20. May 2019.

8062 Scopula imitaria (Hübner, [1799]): Pridvorica, 13. May 2018., 1f. Rebel was (1917) first who reported this species for Metohia.

8241 Scotopteryx luridata (Hufnagel, 1767): Pasjane, 22. April 2018., 1f.
8269 Catarhoe cuculata (Hufnagel, 1767): Pridvorica, 7. May 2018., 1f.

8309 Earophila badiata ([Denis \& Schiffermüller], 1775): Šrpce, 29. April 2019., 8m.

8310 Anticlea derivata ([Denis \& Schiffermüller], 1775): Pridvorica, 15. April 2018.,1f.

8335 Gandaritis pyraliata ([Denis \& Schiffermüller], 1775): Gornje Kusce, 13. June 2018., $1 \mathrm{f}$.

8338 Ecliptopera silaceata ([Denis \& Schiffermüller], 1775): Štrpce, 20. May 2019., $1 \mathrm{~m}$.

8341 Chloroclysta siterata (Hufnagel, 1767): Šar-Planina, Štrpce, 890 m, 20. May 2019., 1f. Genitalia checked, slide SR-3123.

8400 Horisme vitalbata ([Denis \& Schiffermüller], 1775): Pridvorica, 10. May 2018., 1m; Štrpce, 20. May, $1 \mathrm{~m}$.

8411 Melanthia procellata ([Denis \& Schiffermüller], 1775): Pridvorica, 5-10. May 2018., 3m; 20. May - 6. June 2018., 6m; Šar-Planina, Štrpce, 20. May 2019. (Fig. 9).

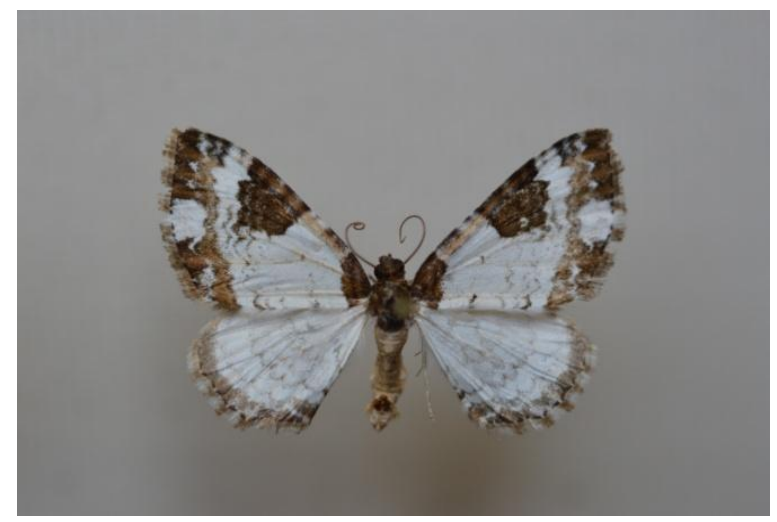

Figure 9. M. procellata ([Denis \& Schiffermüller], 1775) ŠarPlanina, Štrpce, 890 m, 20. May 2019., Female.

8447 Operophtera brumata (Linnaeus, 1758): Vrbeštica, 23 December 2018., $7 \mathrm{~m}$.

8622 Aplocera efformata (Guené, [1858]): Štrpce, 9. June 2018. $1 f$.

\section{DISCUSSION AND CONCLUSION}

Presented here are the 48 species of the Geometridae Leach, 1815 family recorded in Kosovo and Metohia. The specimens were collected from 15 localities during winter and spring in 1979., 1980., 1982., 2018. and 2019.

Five species are reported as new for the fauna of Kosovo and Metohia: Plagodis pulveraria (Linnaeus, 1758), Scotopteryx luridata (Hufnagel, 1767), Catarhoe cuculata (Hufnagel, 1767), Earophila badiata (Denis\&Schiffermüller, 1775) and Anticlea derivata (Denis \& Schiffermüller, 1775).

Special attention was paid to the genus Scopula Schrank, 1802. Sihvonen (2005) treated the drenowskii Sterneck, 1941 taxon as a subspecies of decorata ([Denis \& Schiffermüller], 1775). However, Can (2009) proved that this taxon is a valid 
species (bona species). Alphéraky (1876) described the orientalis, which has recently been found in Romania by Dinca \& Szekely (2018). Since the original description was given in Russian, we kindly asked Dr Konstantin Efetov (Simferopol, Russia) to provide us with a translation which we present here:

«-Decorata Brkh. Specimens from Taganrog are strongly lighter than German ones; transverse lines crossing middle of wings are slightly visible; lace-shaped pattern near outer margin is much more pale and does not rich upper angle of forewing. Size of specimens from Taganrog is similar to those of Northern Iran: 27-29mm, while German specimens: 20-23mm. According to my information specimens of this species are larger and lighter in the East than type from Western Europe and I propose to name the eastern form for example as varietas Orientalis. Moth is not rare; flying from end of May till September.»

Among the specimens examined in Kosovo and Metohia, 5 species of the Scopula Schrank, 1802 genus were found, but not the two aforementioned species.

Members of the Geometridae family are particularly sensitive to industrial pollution. This is why melanistic forms occur. Among the specimens collected, we found no melanistic forms. This serves to show that the environment is relatively well preserved.

\section{ACKNOWLEGMENTS}

We would like to thank to all colleagues who kindly provided the material for this study: Janićijević, Tanja (Gnjilane), Makić Mirjana (Gnjilane), Maksimović Milica (Gnjilane), Mladenović, Ilija (Štrpce), Orlović, Aleksandar (Štrpce), Raćićević Tijana (Brezovica), Timotijević Mirjana (Leposavić) and Živković, Mirjana (Zubin Potok).

We would like to express our gratitude to Dr. Peder Skou (Ollerup, Denmark), Dr Konstantin Efetov (Simferopol, Crimea) and Dr Stoyan Beshkov (Sofia, Bulgaria) for his valuable contributions.

\section{REFERENCES}

Alphéraky, S. 1875. Cheshuekrylyya (Lepidoptera) okrestnostei Taganroga / The Butterflies (Lepidoptera) of the environs of Taganrog. Russkago Entomologicheskago obshchestva, 8: 150-226 (1875); 10: 35-53 (1876); 11: 45-50 (1878).

Beshkov, S. 2015. Eight new and some rare for Serbia nocturnal Lepidoptera species collected at light. Entomologist's Rec. J. Var., 127, pp. 212-227; Pl. 10.

Bishop, J. A. 1972. An Experimental Study of the Cline of Industrial Melanism in Biston betularia (L.) (Lepidoptera) between Urban Liverpool and Rural North Wales. The Journal of Animal Ecology, 41(1). doi:10.2307/3513

Can, F. 2009. DNA barcoding confirms species rank for a cryptic geometrid species from Turkey and Bulgaria (Lepidoptera: Geometridae: Sterrhinae). Zootaxa, 2314(1), pp. 63-68. doi:10.11646/zootaxa.2314.1.4
De Prins, W. 2016. Catalogue of the Lepidoptera of Belgium: Vlaamse Vereniging voor Entomologie. In Entomobrochure. 9, 1278.

Dincă, V., \& Székely, L. 2018. First record of Scopula orientalis (Alphéraky, 1876) (Lepidoptera, Geometridae) in Romania, at the northern limit of the BalkanS. Nota Lepidopterologica, 41(2), pp. 189-197. doi:10.3897/nl.41.24316

Djurić, M., \& Hric, B. 2013. On distribution area of Asovia maeoticaria (Alphéraky, 1876) (Insecta: Lepidoptera: Geometridae). ZooNotes, 49, pp. 1-2.

Dodok, I. 2006. The fauna of Geometridae (Lepidoptera) in the region of Užice in Western Serbia. Acta entomologica serbica, 11(1/2), pp. 61-75.

Đorović, Đ. 1974. Defolijatori hrasta iz reda Lepidoptera na Kosovu. Beograd: Šumarski fakultet. Magistarski rad.

Đorović, Đ. 1976. Results of observations on the occurrence and morphological characteristics of harmful caterpillars from fam. Geometridae in the oak forests of the Province Kosovo. Šumarski list, 100, pp. 495-501.

Djorović, Dj., 1979. Fauna leptira (Lepidoptera) Metohije za period 1977-1978. god. - Report (unpublished), pp. 1-17. Peć.

Đorović, Đ. 1980. Karakteristike i struktura biocenotskog kompleksa defolijatora iz reda Lepidoptera $\mathrm{u}$ hrastovim šumama Kosova. Beograd: Šumarski fakultet. Doktorska disertacija.

Đorović, Đ. 1992. Biocenotički kompleks gusenica hrasta (Biocoenotis's complex of Oak's tree caterpillars). Nauka i društvo 1-191; 27 tabsl 67 figs.

Erlacher, S-I. 1998. Die phylogenetischen Beziehungen innerhalb des Taxon Dyscia Hübner, (Lepidoptera: Geometridae). Jena: Friedrich-Schiller-Universität, Jena Biologisch-Pharmazeutische Fakultät, Institut für Spezielle Zoologie und Evolutionsbiologie mit Phyletischem Museum. 1-105. Diplomarbeit.

Ford, E.B. 1955. Moths. London: Collins.

Jakšić, P. 2016. New contributions to the knowledge of Lepidortera fauna of Kosovo and Metohia (Republic of Serbia). The University Thought - Publication in Natural Sciences, 6(2), pp. 1-4. doi:10.5937/univtho6-12528

Jakšić, P., \& Ristić, G. 1999. 2001. New and rare species of Lepidoptera in Yugoslavia. Acta entomologica serbica, Beograd, 4(1/2), pp. 63-74.

Karsholt, O., \& Razowski, J. 1996. The Lepidoptera of Europe. Apollo Books.

Müller, B., Erlacher, S., Hausmann, A., Rajaei, H., Sihvonen, P., Skou, P., 2019. The Geometrid Moths of Europe, 6(1\&2), Leiden; Boston: Brill.

Van Nieukerken, E., et al., 2011. Order Lepidoptera. In Z. ZhiQiang Ed., Animal biodiversity: An outline of higher-level classification and survey of taxonomic richness: Zootaxa. 3148, pp. 1-237.

Pinker, R. 1968. Die Lepidopterenfauna Mazedoniens. III. Geometridae. Prirodonaučen Muzej Skopje, 4, pp. 1-72.

Rebel, H. 1917. Lepidopteren aus Neumontenegro. Mathematisch-naturwissenschaftliche Klasse, 126: 765-813; Abteilung 1; Kaiserliche Akademie der Wissenschaften in Wien.

Ristić, G. 1997. Contribution to Knowledge of Distribution of Species Genus Aplocera Stephens, 1827 in Serbia 
(Lepidoptera, Geometridae). University Thought, Nat. Sci., III: 41-43; 3 figs.

Sihvonen, P. 2005. Phylogeny and classification of the Scopulini moths (Lepidoptera: Geometridae, Sterrhinae). Zoological Journal of the Linnean Society, 143(4), pp. 473-530. doi:10.1111/j.1096-3642.2005.00153.x

Stojanović, A., Jovanović, M., \& Marković, Č. 2018. Interesting species of the Family Geometridae (Lepidoptera) recently collected in Serbia, including some that are new to the country's fauna. Acta entomologica serbica: Color tabs., 23(2): 27-41; 8 color tabs.
Tomić, D., Zečević, M., Mihajlović, Lj., \& Glavendekić, M. 2002. Fauna zemljomerki (Lepidoptera, Geometridae) Srbije. In Zbornik radova o fauni Srbije. Beograd: SANU. Knjiga VI: 73-164.

Urbahn, E. 1966. Ergebnisse der Albanien-Expedition 1961 des Deutschen Entomologischen Institutes. 51. Beitrag Lepidoptera: Geometridae. Beiträge zur Entomologie, 16(3/4), pp. 407-446.

Vukčević, R. 1954. Biljne štetočine i bolesti utvrđene na Kosmetu od 1949-1954 godine. Zaštita bilja, 26, pp. 56-106. 